\title{
60-jährige Asthma-Patientin mit Dyspnoe nach bronchialer Thermoplastie
}

\author{
A 60-Year-Old Patient with Asthma and Dyspnoea after Bronchial \\ Thermoplasty
}

\section{Autoren}

Dirk Skowasch ${ }^{1}$, Johanna Klein ${ }^{1}$, Rami Homsi²,

Carmen Pizarro ${ }^{1}$

Institut

1 Medizinische Klinik und Poliklinik II - Sektion Pneumologie

2 Radiologische Klinik; Universitätsklinikum Bonn
Bibliografie

DOI https://doi.org/10.1055/s-0043-123059

Dtsch Med Wochenschr 2018; 143: 381-382

(c) Georg Thieme Verlag KG, Stuttgart · New York ISSN 0012-0472

Korrespondenzadresse

Prof. Dr. Dirk Skowasch

Medizinische Klinik und Poliklinik II, Sektion Pneumologie,

Universitätsklinikum Bonn, Sigmund-Freud-Str. 25,

53127 Bonn

dirk.skowasch@ukbonn.de
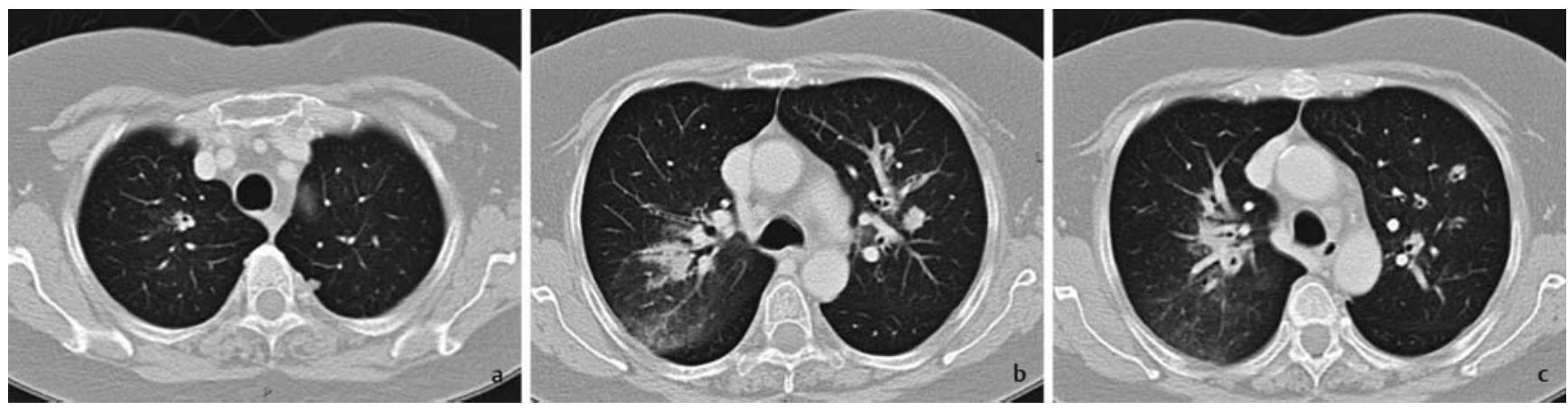

- Abb. 1 a-c Thorax-CT (transversal, Kontrastmittel-verstärkt) 3 Tage nach bronchialer Thermoplastie.

Bei einer 60-jährigen Patientin besteht seit Jahren ein schweres allergisches Asthma bronchiale. Trotz Ausschöpfen aller Therapieoptionen inklusive Omalizumab bestand keine ausreichende Symptomkontrolle. Andere Differenzialdiagnosen oder Komorbiditäten waren im Vorfeld - auch durch Computertomografie (CT) des Thorax - ausgeschlossen worden; Bronchiektasen lagen nicht vor. Daher erfolgte vor 6 und 12 Wochen in 2 Sitzungen die unkomplizierte bronchiale Thermoplastie der beiden Unterlappen. Direkt nach der 3. Behandlung mit 80 Energieabgaben in beiden Oberlappen (Alair-System, Firma Boston Scientific, Vollnarkose, Dauer 30 Minuten) entwickelte die Patienten Dyspnoe. Laborchemisch ergab sich keine Infektkonstellation, mikrobiologisch kein Keimnachweis im Trachealsekret. 3 Tage nach der bron- choskopischen Intervention wurde die vorliegende CT-Aufnahme des Thorax angefertigt. Sie zeigt 3 pathologische Befunde $(\triangleright$ Abb. 1).

\section{FRAGEN}

- Welche Befunde sind es?

- Erlauben diese Befunde eine Diagnose? Wenn ja, welche?

- Sind Differenzialdiagnosen möglich? Wenn ja, welche? 


\title{
60-jährige Asthma-Patientin mit Dyspnoe nach bronchialer Thermoplastie
}

\author{
A 60-Year-Old Patient with Asthma and Dyspnoea after Bronchial \\ Thermoplasty
}
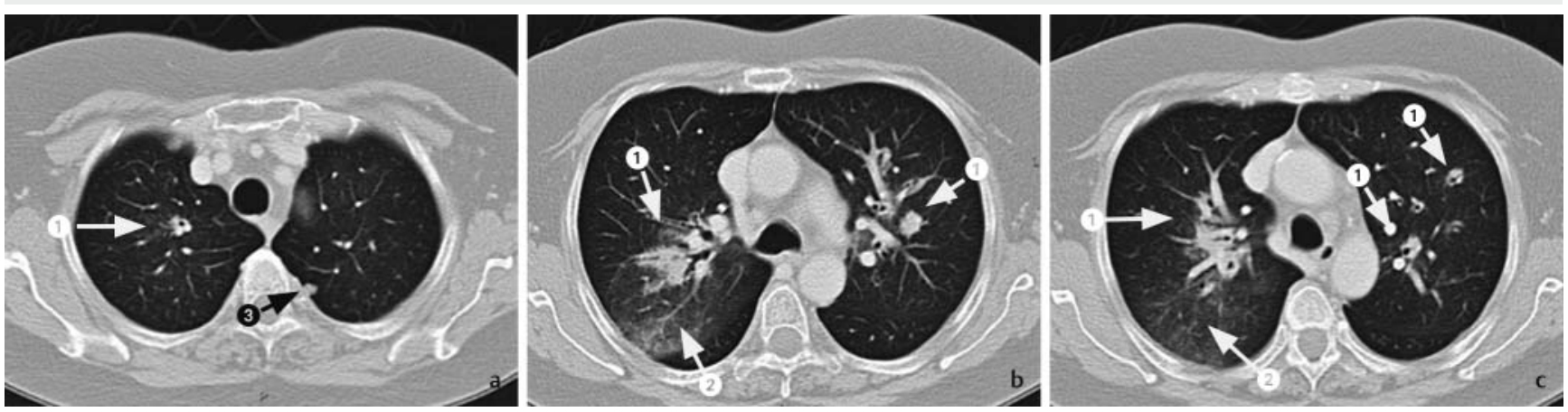

- Abb. 2 a-c Thorax-CT (transversal, Kontrastmittel-verstärkt) 3 Tage nach bronchialer Thermoplastie und d 3 Monate nach bronchialer Thermoplastie.

\section{Befunde}

1. peribronchiale Konsolidierungen in den behandelten Subsegmenten beider Oberlappen

2. Milchglas in den umliegenden Bereichen

3. Rundherd paravertebral und pleuraständig $7 \times 7 \mathrm{~mm}$ im linken Oberlappen

\section{Diagnose}

Postinterventionelle Konsolidierungen nach bronchialer Thermoplastie beider Oberlappen. Inzidenteller Lungenrundherd.

\section{Differenzialdiagnose}

Infektiöse Pneumonie.

\section{Erläuterung}

Die bronchiale Thermoplastie ist ein interventionell-bronchoskopisches Verfahren zur Reduktion der glatten Muskulatur der Bronchialwände, wodurch es die Bronchialverengung bei schwerem Asthma bronchiale verringern soll. Es ist im Hinblick auf Reduktion von Exazerbationen, Notfallvorstellungen und Steroidbedarf sowie Verbesserung der Lebensqualität etabliert. Die wichtigste Komplikation ist ein durch die Prozedur induzierter Asthmaanfall. Im vorliegenden Fall finden sich 3 Tage nach Thermoplastie typische Veränderungen, wie sie erst kürzlich als transiente Reaktion auf die RadiofrequenzBehandlung beschrieben wurden [1]. Auch im vorliegenden Fall waren die Veränderungen nur passager (CT-Verlaufskontrolle des stabilen Rundherds nach 3 Monaten). Die Patientin hat nun, 1/2 Jahr nach Therapie, deutlich profitiert. In Kenntnis der typischen und transienten bildgebenden Veränderungen im Kontext mit o.g. laborchemischen und mikrobiologischen Befunden sind unnötige Antibiotika-Gaben zu vermeiden.

\section{Literatur}

[1] Debray MP, Dombret MC, Pretolani M et al. Early computed tomography modifications following bronchial thermoplasty in patients with severe asthma. Eur Resp J 2017; 49: 1601565 